\title{
ON THE PRIMITIVITY OF POLYNOMIAL RINGS WITH NONPRIMITIVE COEFFICIENT RINGS
}

\author{
T. J. HODGES
}

\begin{abstract}
For a hereditary noetherian prime ring $R$ with classical quotient ring $Q$, various necessary and sufficient conditions are given for the polynomial ring $R\left[X_{1}, \ldots, X_{n}\right]$ to be primitive when $R$ itself is not primitive. It is shown that if $R$ is a local hereditary noetherian prime ring, then $R[X]$ is primitive if and only if $Q[X]$ is primitive. Similarly, for a semilocal hereditary noetherian prime ring $R$ whose Jacobson radical contains a nonzero central element, it is shown that $R\left[X_{1}, \ldots, X_{n}\right]$ is primitive if and only if $Q\left[X_{1}, \ldots, X_{n}\right]$ is primitive.
\end{abstract}

1. Introduction. In this paper we investigate which nonprimitive rings can occur as coefficient rings of primitive polynomial rings (primitive will mean right primitive throughout). Whether such rings could exist was an open problem for many years until the first examples were given in $[\mathbf{H}]$. In this article we show that such rings are in fact quite common. In particular, as corollaries to our main results (Theorems 3.3 and 3.5) we show that if $R$ is a hereditary noetherian prime ring (abbreviated to HNP ring) whose (classical) quotient ring is transcendental over its center, then $R[X]$ is primitive if either of the following additional conditions is satisfied: (i) $R$ is local; or (ii) $R$ is semilocal and the Jacobson radical of $R$ contains a nonzero central element. Examples of such rings include the (classical) localisation at a height one semiprime ideal of any of the following rings: (i) the enveloping algebra of a (nonabelian) nilpotent Lie algebra; (ii) the group algebra of a (nonabelian) nilpotent polyinfinite-cyclic group; (iii) the ring of polynomials over a division ring transcendental over its center.

The focus of our attention is on HNP rings. It is easy to show that for such a ring $R$ is nonprimitive precisely if it is bounded (that is, all essential left or right ideals contain a two-sided ideal). In the first section we show that for a bounded HNP ring $R$ with quotient ring $Q$, necessary conditions for $R\left[X_{1}, \ldots, X_{n}\right]$ to be primitive are: (i) $Q\left[X_{1}, \ldots, X_{n}\right]$ is primitive; and (ii) $R$ is semilocal. This immediately restricts our range of study considerably. (It should be recalled at this stage that in a recent generalization of an old result due to Jacobson, Amitsur and Small showed in [AS], that for a simple artinian ring $Q, Q\left[X_{1}, \ldots, X_{n}\right]$ is primitive if and only if for some $s$, the $s \times s$ matrix ring $M_{s}(Q)$ has transcendence degree at least $n$ over its center.) In the second section we show that in some situations these conditions are sufficient. In particular, we prove that if the Jacobson radical of $R$ contains a nonzero central element, then $R\left[X_{1}, \ldots, X_{n}\right]$ is primitive if and only if $Q\left[X_{1}, \ldots, X_{n}\right]$ is primitive.

The above results, together with the fact that the examples given in $[\mathbf{H}]$ are all local, tend to suggest that a necessary condition for a nonprimitive ring to be the coefficient ring of a primitive polynomial ring is that $R$ must be semilocal. In the

Received by the editors July 29, 1985.

1980 Mathematics Subject Classification (1985 Revision). Primary 16A20.

Key words and phrases. Primitivity, polynomial rings, hereditary noetherian prime rings. 
last section we show that this is false by constructing a ring $R$ satisfying: (i) $R$ is not primitive; (ii) $R$ is semiprimitive; and (iii) $R[X]$ is primitive.

2. Some necessary conditions. Our first result is probably well known. We include it for the sake of completeness.

PROPOSITION 2.1. Let $R$ be a bounded noetherian prime ring with quotient ring $Q$. Then any simple faithful $R\left[X_{1}, \ldots, X_{n}\right]$-module is naturally a $Q\left[X_{1}, \ldots, X_{n}\right]$ module. In particular, if $R\left[X_{1}, \ldots, X_{n}\right]$ is primitive, then $Q\left[X_{1}, \ldots, X_{n}\right]$ is primitive.

PROOF. Let $S$ be the set of regular elements of $R$. Then the boundednes of $R$ implies that any simple faithful $R\left[X_{1}, \ldots, X_{n}\right]$-module is $S$-torsion free and $S$-divisible.

LEMMA 2.2. Let $R$ be an HNP ring with infinitely many isomorphism classes of simple modules. Let $a_{1}, \ldots, a_{n}$ be elements of the quotient ring $Q$ of $R$. Then there exists a ring $S$ containing $R$ and strictly contained in $Q$ such that $\left\{a_{1}, \ldots, a_{n}\right\} \subseteq S$.

PROOF. Let $Z$ be the set of isomorphism classes of simple modules which occur as subfactors of $\left(a_{i} R+R\right) / R$ for some $i$. Since $\left(a_{i} R+R\right) / R$ has finite length for all $i, Z$ is finite. Let $S$ be the localisation described in [S, VIII.3 and IX] or $[\mathbf{G}]$ with respect to the torsion theory generated by the simple modules of $Z$. Then $\left\{a_{1}, \ldots, a_{n}\right\} \subseteq S$. Further, by $[\mathbf{G}$, Theorem 3], $S$ also has infinitely many isomorphism classes of simple modules, so $S$ is strictly contained in $Q$.

THEOREM 2.3. Suppose that $R$ is a bounded HNP ring such that $R\left[X_{1}, \ldots, X_{n}\right]$ is primitive for some $n \geq 1$. Then $R$ is semilocal.

Proof. Let $Q$ be the simple artinian quotient ring of $R$ and let $V$ be a simple faithful $R\left[X_{1}, \ldots, X_{n}\right]$-module. Then by Proposition $2.1, V$ is a simple $Q\left[X_{1}, \ldots, X_{n}\right]$-module. By the Amitsur-Small nullstellensatz [AS], $V$ must have finite length as a $Q$-module. So $V \cong L^{(t)}$, where $L=e Q$ is a simple right ideal of $Q$ and $e$ is idempotent. The action of $X_{1}, \ldots, X_{n}$ on $V$ can then be identified with left multiplication by elements $\tau_{1}, \ldots, \tau_{n} \in M_{t}(e Q e)$. Suppose that $R$ is not semilocal and use Lemma 2.1 to find a ring $S$ properly contained in $Q$ and containing $R, e$ and all the coefficients of the matrices $\tau_{1}, \ldots, \tau_{n}$. Let $I=L \cap S=e S$. Since $S$ is strictly contained in $Q, I$ must be strictly contained in $L$. Since all the coefficients of the $\tau_{i}$ lie in $S$, it is clear that $I^{(t)}$ must be a proper, nonzero $R\left[X_{1}, \ldots, X_{n}\right]$-submodule of $V$, contradicting the simplicity of $V$.

REMARK. It is perhaps pertinent to point out that Theorem 2.3 is nontrivial in the sense that there do exist bounded HNP rings whose quotient divison rings are transcendental over their center and which are not semilocal. For example, let g be a nonabelian solvable Lie algebra over the complex numbers and let $U$ be the enveloping algebra of $\mathbf{g}$. It is proved in [C, Theorem 3.3] that the intersection $T$ of the localisations of $U$ at height-one prime ideals is a bounded hereditary noetherian domain. In general, $T$ may be semilocal; however, if $\mathbf{g}$ is nilpotent, then $T$ has infinitely many maximal ideals and hence is not semilocal.

3. Some sufficient conditions. We now proceed to show that if the necessary conditions given in Proposition 2.1 and Theorem 2.3 are strengthened a little, they become sufficient. In the first case (Theorem 3.3) we assume that the Jacobson 
radical of $R$ contains a nonzero central element. In the second case (Corollary 3.6) we assume that $R$ is local (although here we are only able to conclude that $R\left[X_{1}\right]$ is primitive). It seems likely that the conditions of Proposition 2.1 and Theorem 2.3 are not in themselves sufficient; however, we have been unable to construct a suitable counterexample.

PROPOSITION 3.1. Let $R$ be a semilocal HNP ring with Jacobson radical $J$ and quotient ring $Q$. Suppose that $V$ is a simple faithful $Q\left[X_{1}, \ldots, X_{n}\right]$-module containing a nonzero element $v$ annihilated by $1+\delta$, where $\delta \in J R\left[X_{1}, \ldots, X_{n}\right]$. Then $V$ is a simple faithful $R\left[X_{1}, \ldots, X_{n}\right]$-module.

PROOF. Let $W=v R\left[X_{1}, \ldots, X_{n}\right]$, let $I_{0}=\operatorname{ann}_{Q\left[X_{1}, \ldots, X_{n}\right]}(v)$ and let $I=I_{0} \cap$ $R\left[X_{1}, \ldots, X_{n}\right]$, so $W \cong R\left[X_{1}, \ldots, X_{n}\right] / I$. Because two-sided ideals in $R\left[X_{1}, \ldots, X_{n}\right]$ blow up to two-sided ideals in $Q\left[X_{1}, \ldots, X_{n}\right], W$ must be faithful. If $W$ is not simple, then there exists a maximal right ideal $L$ of $R\left[X_{1}, \ldots, X_{n}\right]$ strictly containing $I$. Since $I_{0}$ is maximal, $L Q\left[X_{1}, \ldots, X_{n}\right]=Q\left[X_{1}, \ldots, X_{n}\right]$, and so $L$ contains a regular element of $R$. Since $R$ is hereditary, it follows that $R / L$ has finite length and hence that $L$ contains some power of $J$. Pick $s$ to be the smallest positive integer such that $J^{s} \subseteq L$. For all $p \in J^{s-1}$, since $1+\delta \in L$, it follows that $(1+\delta) p \in L$. On the other hand, the hypothesis on $\delta$ implies that

$$
\delta p \in J R\left[X_{1}, \ldots, X_{n}\right] J^{s-1}=J^{s} R\left[X_{1}, \ldots, X_{n}\right] \subseteq L .
$$

Thus $p \in L$ for all $p \in J^{s-1}$, contradicting the choice of $s$.

Hence $W$ is a simple faithful $R\left[X_{1}, \ldots, X_{n}\right]$-module. So by Proposition 2.1, $W$ is a $Q\left[X_{1}, \ldots, X_{n}\right]$-submodule of $V$, whence $W=V$.

COROLlARY 3.2. Let $R$ be a semilocal hereditary noetherian domain with Jacobson radical $J$ and quotient division ring $D$. Let $F$ be the centre of $D$ and suppose that $J$ contains an element $p$ transcendental over $F$. Then $R[X]$ is primitive. Moreover, if $p$ is contained in a subfield of transcendence degree $n$ over $F$, then $R\left[X_{1}, \ldots, X_{n}\right]$ is primitive.

PROOF. Let $p_{1}=p$ and let $F\left(p_{1}, \ldots, p_{n}\right)$ be a purely transcendental extension field of $F$ lying inside $D$. Make $D$ into a $D\left[X_{1}, \ldots, X_{n}\right]$-module $V$ by letting $X_{i}$ act via left multiplication by $p_{i}^{-1}$. Then $V$ is clearly a simple faithful $D\left[X_{1}, \ldots, X_{n}\right]-$ module. But the element $1 \in V$ is annihilated by $1-p_{1} X_{1}$, so $V$ is a simple faithful $R\left[X_{1}, \ldots, X_{n}\right]$-module by the proposition.

THEOREM 3.3. Let $R$ be a semilocal HNP ring with quotient ring $Q$. Suppose that the Jacobson radical of $R$ contains a nonzero central element. Then the following conditions are equivalent.

(i) $R\left[X_{1}, \ldots, X_{n}\right]$ is primitive.

(ii) $Q\left[X_{1}, \ldots, X_{n}\right]$ is primitive.

(iii) For some positive integer $t, M_{t}(Q)$ has transcendence degree over its centre at least $n$.

PROOF. (ii) $\Leftrightarrow$ (iii) is a trivial generalisation of Amitsur and Small's result for division rings [AS, Theorem 2].

(i) $\Rightarrow$ (ii) follows from Proposition 2.1. 
(ii) $\Rightarrow$ (i) Let $D$ be a divison ring such that $Q \cong M_{s}(D)$ for some $s$, and let $F$ be the centre of $D$. Then by a trivial generalisation of Amitsur and Small's nullstellensatz for division rings $\left[\mathbf{A S}\right.$, Theorem 1], any simple $Q\left[X_{1}, \ldots, X_{n}\right]$-module has finite length over $Q$. Let $V$ be a simple faithful $Q\left[X_{1}, \ldots, X_{n}\right]$-module. Then, in particular, $V$ is finite dimensional over $D$. Let $v$ be a nonzero element of $V$ and let $I_{0}$ be the annihilator in $Q\left[X_{1}, \ldots, X_{n}\right]$ of $v$. Then $I_{0}$ contains a polynomial of the form

$$
1+a_{1} X_{1}+\cdots+a_{d} X_{1}^{d} \quad \text { where } a_{1}, \ldots, a_{d} \in D
$$

Let $J$ be the Jacobson radical of $R$. By hypothesis we may pick a nonzero $p \in F \cap J$. Since $R$ is hereditary, there exists a positive integer $r$ such that $p^{r} a_{i} \in J$ for all $i=1, \ldots, d$. Let $\phi$ be the $Q$-linear automorphism of $Q\left[X_{1}, \ldots, X_{n}\right]$ given by

$$
X_{1} \rightarrow p^{r} X_{1}, X_{2} \rightarrow X_{2}, \ldots, X_{n} \rightarrow X_{n} .
$$

If $I=\phi\left(I_{0}\right)$, then $I$ is also an unbounded maximal right ideal of $Q\left[X_{1}, \ldots, X_{n}\right]$. Further, $I$ contains the element $1+a_{1} p^{r} X_{1}+\cdots+a_{d} p^{r d} X_{1}^{d}$. Since all the $a_{i} p^{r i}$ lie in $J$, Proposition 3.1 implies that $R\left[X_{1}, \ldots, X_{n}\right]$ is also primitive.

In particular, under the hypotheses of the theorem, $R$ has the following property: If $R\left[X_{1}, \ldots, X_{n}\right]$ is primitive, then $R\left[X_{1}, \ldots, X_{m}\right]$ is primitive for all $m \leq n$. It is an interesting open question whether this property holds for all rings.

EXAMPLE 3.4. Let $\mathbf{g}$ be a nilpotent Lie algebra over a field $k$ of characteristic zero and let $U$ be the enveloping algebra of $\mathbf{g}$. Then $U$ has a quotient division ring $D$ which is isomorphic to the quotient division ring of the $n$th Weyl algebra over some transcendental extension field of $k$, where $n$ is a positive integer depending on $\mathbf{g}[\mathbf{D}, 4.7 .18]$.

It is well known that all ideals of $U$ have centralising sets of generators $[\mathbf{M}]$ and hence that any semiprime ideal is localisable. Let $R$ be a localisation of $U$ at the intersection of a finite set of height one primes. Then $R$ is a semilocal hereditary noetherian domain whose Jacobson radical contains a central element. Now it is shown in $[\mathbf{A S}]$ that $D\left[X_{1}, \ldots, X_{t}\right]$ is primitive if and only if $t \leq n$. Hence by the theorem $R\left[X_{1}, \ldots, X_{t}\right]$ is primitive if and only if $t \leq n$.

It would obviously be nice to be able to remove the hypothesis in Theorem 3.3 that the Jacobson radical contain a central element, at least in the case when $n=1$. Unfortunately we cannot, in general, do this; the main obstacle is that we do not know whether the centre of $Q$ is necessarily algebraic over the quotient field of the centre of $R$. For this reason we restrict ourselves to a special kind of semilocal HNP ring.

Recall that an $R$-ideal is defined to be an $R$-subbimodule of $Q$. An $R$-ideal $I$ is said to be invertible if $I I^{*}=I^{*} I=R$ for $I^{*}=\{x \in Q \mid x I \subseteq R\}$. It is well known that for an hereditary noetherian prime ring $R$, the invertible $R$-ideals form a group which will be infinite cyclic if either (i) $R$ is local or (ii) $R$ is semilocal and the maximal ideals of $R$ are idempotent and form a single cycle (see [ER] for details).

THEOREM 3.5. Let $R$ be a semilocal hereditary noetherian domain with quotient division ring $D$. Suppose that the group of invertible ideals of $R$ is cyclic. Then $R[X]$ is primitive if and only if $D[X]$ is primitive.

PROOF. By Proposition 2.1 it suffices to show that if $D[X]$ is primitive, then $R[X]$ is primitive. Let $J$ be the Jacobson radical of $R$ and let $F$ be the centre of $D$. 
If $J \cap F \neq 0$, then the result follows from Theorem 3.3. So assume $J \cap F=0$. For any nonzero $f$ in $F$, the module $f R$ is an invertible $R$-ideal. If $F$ is not contained in $R$, there exists an $f$ such that $f R \neq R$. In this case, any ideal containing an invertible ideal contains $f^{n} R$ for some integer $n$. In particular, this is true for the Jacobson radical $J$, contradicting the assumption that $J \cap F=0$. Hence $F \subseteq R$, and it is then easy to see that all nonzero elements of $J$ must be transcendental over $F$. The result then follows from Corollary 3.2 .

COROLlaRY 3.6. Let $R$ be a local HNP ring with quotient ring $Q$. Then $R[X]$ is primitive if and only if $Q[X]$ is primitive.

PROOF. A local HNP ring is a complete matrix ring over a local hereditary neotherian domain $[\mathbf{C H}, 10.8]$. The result then follows from the fact that $T$ is primitive if and only if $M_{n}(T)$ is primitive.

4. A semiprimitive example. The results of the previous two sections and the examples given in $[\mathbf{H}]$ tend to suggest that nonprimitive rings $R$, such that $R[X]$ is primitive, must be semilocal. We now show that this is not the case by giving an example of such a ring which is semiprimitive.

Let $D$ be a division ring with centre $F$, and let $T=D[Y, Z]$ be the polynomial ring over $D$ in two commuting indeterminates. Let $P=Y T+Z T$, and let $S_{0}=$ $T \backslash P$. Since $P$ is generated by central elements, $S_{0}$ is a left and right Ore set by [M, Theorem 6]. Similarly in $D[Z]$, the complement of the ideal $Z D[Z]$ is a left and right Ore set. We denote the corresponding localisation $D[Z]_{(Z)}$.

LEMMA 4.1. Let $A=D[Z]_{(Z)}[Y]$, and let $B$ be a left and right Ore localisation of $T$ contained between $A$ and $T_{S_{0}}$. If $D$ has transcendence degree at least two over its centre, then $B[X]$ is primitive.

PROOF. Let $C$ be the centre of $D$, and let $C\left(t_{1}, t_{2}\right)$ be a subfield of $D$ of transcendence degree two over $C$. We first construct a simple faithful $A[X]$-module. Let $M$ be the division ring $D(Z)$ (the quotient division ring of $D[Z]$ ) considered as a $D[Z]_{(Z)}$-module via right multiplication and on which $Y$ acts via left multiplication by $t_{1} Z$. This makes $M$ into a well-defined $A$-module, and it is clear that $M$ has a unique chain of submodules

$$
M \supseteq \cdots \supseteq Z^{i} A \supseteq Z^{i+1} A \supseteq \cdots \supseteq 0 .
$$

Hence if we let $X$ act on $M$ as the $A$-module endomorphism given by left multiplication by $t_{2} Z^{-1}$, this makes $M$ into a simple $A[X]$-module. To see that $M$ is faithful, first note that any two-sided ideal of $A[X]$ contains an element of $C[Y, Z, X]$. However, by assumption on $t_{1}$ and $t_{2}$, the endomorphisms of $M$ given by left multiplication by $t_{1} Z, Z$ and $t_{2} Z^{-1}$ are clearly algebraically independent. Thus $M$ is a simple faithful $A[X]$-module.

Now observe that if $1_{M}$ is the unit element of $D(Z)$ as an element of $M$, then $\operatorname{ann}_{T}\left(1_{M}\right)=\left(Y-t_{1} Z\right) T \subseteq Y T+Z T$. Hence $S_{0} \cap \operatorname{ann}_{T}\left(1_{M}\right)=\varnothing$. Thus $M \otimes_{A[X]}$ $T_{S_{0}}[X] \neq 0$, so $M \otimes_{A[X]} B[X] \neq 0$ also. Hence by standard localisation theory, since $B[X]$ is an Ore localisation of $A[X]$, we must have that $M \otimes_{A[X]} B[X]$ is a simple faithful $B[X]$-module.

Now let $P_{\lambda}=(Y+\lambda) T$ for each $\lambda \in F$. Let $\Lambda$ be a (possibly infinite) subset of $F \backslash\{0\}$, and let $S=T \backslash\left(P \cup \bigcup_{\lambda \in \Lambda} P_{\lambda}\right)$. 
LEMMA 4.2. S is a left and right Ore set in $T$.

Proof. Let $S_{0}=T \backslash P$. Recall that $S_{0}$ is an Ore set. Clearly $S \subseteq S_{0}$, so for all $r \in T$ and $s \in S$ there exist $r^{\prime} \in T, s^{\prime} \in S_{0}$ such that $r s^{\prime}=s r^{\prime}$. By picking $s^{\prime}$ of smallest total degree in $Y$ and $Z$, we may assume that $s^{\prime}$ and $r^{\prime}$ have no common central factors. Suppose that $s^{\prime} \in P_{\lambda}$ for some $\lambda \in \Lambda$. Then $s r^{\prime}=r s^{\prime} \in P_{\lambda}$. Since $s \notin P_{\lambda}$ and $P_{\lambda}$ is completely prime, $r^{\prime}$ must also belong to $P_{\lambda}$. But then $Y+\lambda$ is a common central factor of both $s^{\prime}$ and $r^{\prime}$. The Ore condition on the other side follows similarly.

THEOREM 4.3. Let $R=T_{S}$. Suppose that $D$ has transcendence degree at least two over its centre, and suppose that $\Lambda$ is an infinite set. Then

(i) $R$ is not primitive,

(ii) $R$ is semiprimitive,

(iii) $R[X]$ is primitive.

ProOF. Firstly, notice that any irreducible polynomial in $T$ with nonzero constant term and not of the form $Y+\lambda$ for some $\lambda \in \Lambda$ lies in $S$ and hence is invertible in $R$. It then follows easily that the ideals $P_{\lambda} R$ of $R$ are maximal. Since the intersection of the $P_{\lambda} R$ is zero, part (ii) follows immediately.

It also follows from the first remark that any element of $R$ not contained in $P R$ is of the form $c u$, where $c$ is central and $u$ is a unit. Hence any unbounded right (or left) ideal must be contained in $P R$ and is therefore not maximal. So $R$ is not primitive.

Part (iii) follows from Lemma 4.1.

NOTE ADDED IN PROOF. Some of the questions asked in the text have been answered by Resco, Warfield and Stafford in Fully bounded G-rings (to appear in Pacific J. Math.). In particular they show that if $R$ is a bounded HNP ring, then $R[X]$ is primitive if and only if $R$ is semilocal and $Q[X]$ is primitive.

\section{REFERENCES}

[AS] S. A. Amitsur and Lance W. Small, Polynomials over division rings, Israel J. Math. 31 (1978), 353-358.

[C] A. W. Chatters, Non-commutative unique factorisation domains, Math. Proc. Cambridge Philos. Soc. 95 (1984), 49-54.

[CH] A. W. Chatters and C. R. Hajarnavis, Rings with chain conditions, Pitman, London, 1980.

[D] J. Dixmier, Enveloping algebras, North-Holland, Amsterdam, 1977.

[ER] D. Eisenbud and J. C. Robson, Hereditary noetherian prime rings, J. Algebra 16 (1970), 86-104.

[G] K. R. Goodearl, Localisation and splitting in HNP rings, Pacific J. Math. 53 (1974), 137-151.

[H] T. J. Hodges, An example of a primitive polynomial ring, J. Algebra 90 (1984), 217-219.

[M] J. C. McConnell, Localisation in enveloping rings, J. London Math. Soc. 43 (1968), 421428.

[S] B. Stenstrom, Rings, of quotients, Springer-Verlag, Berlin, 1975.

Department of Mathematical Sciences, University of Cincinnati, CincinNATI, OHIO 45221 